\title{
Estrategias de autorregulación y competencia discursiva en el nivel superior
}

\section{Self-regulation strategies and discursive competence at the higher level}

\author{
Fiorella Moreno Milla \\ Universidad Nacional Mayor de San Marcos, Lima, Perú \\ ORCID: https://orcid.org/0000-0003-0504-1795 \\ Jessica Paola Palacios Garay \\ Universidad Nacional Mayor de San Marcos, Lima, Perú \\ ORCID: https://orcid.org/0000-0002-2315-1683 \\ Fernando Esteban Nuñez Vara \\ Universidad de San Martin de Porres, Lima, Perú \\ ORCID: https://orcid.org/0000-0003-4054-9674
}

Received 10-12-20 Revised 11-25-20

Accepted 02-13-21 On line 02-27-21

*Correspondence

Email: fiorella.moreno1@unmsm.edu.pe
Cite as:

(C) Universidad San Ignacio de Loyola, Vicerrectorado de Investigación, 2021. 


\section{Resumen}

A partir de la exploración de estudios previos, teorías y conceptos involucrados, en los siguientes acápites de este artículo de revisión, se pretende manifestar, de forma conceptual y analítica, la temática referida a las estrategias de autorregulación en el marco educativo, a la metacognición y las estrategias de autorregulación, a la competencia comunicativa y la competencia discursiva en el marco educativo, a la competencia discursiva en el texto académico y, finalmente, a las estrategias de autorregulación y la competencia discursiva escrita de discentes universitarios.

Palabras clave: Estrategias de autorregulación; Competencia discursiva; Nivel superior

\section{Summary}

From the exploration of previous studies, theories and concepts involved, in the following sections of this review paper, it is intended to manifest, in a conceptual and analytical way, the theme related to self-regulation strategies in the educational framework, to metacognition and self-regulation strategies, to communicative competence and discursive competence in the educational framework, to discursive competence in the academic text and, finally, to self-regulation strategies and written discursive competence of university students.

Keywords: Self-regulation strategies; Discursive competence; Higher level

\section{Introducción}

Existe una serie de estrategias de aprendizaje enfocadas a optimizar las diferentes competencias que el estudiante adquiere a lo largo de su proceso formativo, una de ellas está categorizada como la serie de estrategias de autorregulación, las cuales se desarrollan en el ámbito del aprendizaje autorregulado y, por ende, del aprendizaje autónomo, y son definidas como acciones o etapas que se pueden resumir en preguntas como "¿qué voy a hacer?, ¿cómo lo voy a hacer? (planeación); ¿qué estoy haciendo?, ¿cómo lo estoy haciendo? (monitoreo y supervisión); ¿qué tan bien o mal lo estoy haciendo? (revisión y evaluación)". (Díaz y Hernández, 2002, p. 247). Este tipo de preguntas permite inferir que aquellas herramientas activan el control sobre las ideas, las acciones, las emociones, entre otros, del discente universitario, con la finalidad de que este alcance, con éxito, su propósito.

En cuanto a la competencia discursiva escrita, manifestada en la redacción de textos por parte de los discentes de nivel superior y definida como "la capacidad que posee el estudiante para diseñar, dirigir, estructurar textos, incluyendo aspectos genéricos como el desarrollo temático, y la coherencia y la cohesión" (Common european framework of reference for languages: learning, teaching, assessment. Companion volume with new descriptors [MCER], 2018 p. 139), se puede indicar que aquella ha sido aprendida de forma progresiva desde niveles básicos hasta niveles avanzados, según el contexto social. Por lo mencionado, se presupone que el estudiante ingresa a la universidad con un desarrollo eficiente de la competencia señalada líneas más arriba y que, por lo tanto, podrá adaptarse a las producciones escritas contextualizadas al campus (Roa, 2013). Sin embargo, hasta nuestras fechas, se percibe cierta preocupación, por parte de investigadores de las distintas disciplinas involucradas, por estudiar en este campo, tal es el caso de Níkleva y Rico (2015), Pardo (2015), Borgobello, Cattoni y Ventura (2017), García y Fierro (2017), y Bastacini y Kupczyszyn (2020), a nivel internacional; Cerna y Silva (2018), Evangelista y Evangelista (2018), Mesías (2018), y Calderón (2019), a nivel nacional. Esta preocupación, en la mayoría d ellos casos, radica en que los estudiantes universitarios presentan una carencia de efectividad en el desarrollo de la competencia discursiva escrita, evidenciada en los distintos textos académicos. 
En los siguientes acápites de este artículo de revisión, se expondrá y se analizará, sobre la base de estudios previos, teorías y conceptos, la temática referida a las estrategias de autorregulación en relación con la competencia discursiva escrita en el nivel superior.

\section{Las estrategias de autorregulación en el marco educativo.}

Las estrategias de autorregulación se enmarcan dentro del aprendizaje autorregulado, el cual surge, dentro del campo educativo, a partir de los años 80 (Hernández y Camargo, 2017) y se define como aquella práctica educativa en la que el discente es el único directivo de la adquisición de conocimientos y experiencias, los que, a lo largo de su etapa formativa, serán consolidados con las estrategias específicas y necesarias para emplearlos en sus actividades universitarias como en su vida cotidiana (Zimmerman, 1989). Asimismo, se destaca que aquellas técnicas calzan dentro del modelo constructivista de la educación, el cual suscribe al estudiante como constructor de sus propios saberes mediante la estructuración de sus procesos mentales (Flórez, 1994). Es por ello por lo que el estudiante, al utilizarlas de forma idónea, será capaz de construir nuevos significados a partir de los que ya contempla en su mente, conocidos también como conocimientos previos (Wolters, Pintrich y Karabenick, 2003).

Es considerable resaltar que este tipo de estrategias están formadas por un conjunto de fases, operaciones o actividades que se emplean antes, durante y después de la realización de una tarea académica, y se refieren a la planificación (fase 1), supervisión (fase 2) y evaluación (fase 3). La primera operación, conocida como planificación, planeación o forethought, se emplea antes de iniciar una determinada tarea y está constituida por el bosquejo primario y orientador en cuanto a las estrategias y herramientas que van a ser utilizadas para el cumplimiento de los propósitos, así como de las expectativas frente la ejecución completa de la tarea. La segunda operación, conocida como supervisión, control o performance, se utiliza durante el desarrollo de la actividad y guarda relación con la observación continua del desarrollo, ejecución y estructuración de aquella actividad, así como la verificación del cumplimiento de las metas planteadas inicialmente. La tercera operación, conocida como evaluación, revisión o self-reflection, se aplica al finalizar la tarea y concierne a aquel juicio de valor sobre la ejecución general del proyecto, en el que el estudiante se cerciora acerca de la eficacia de los recursos de aprendizaje utilizados (Zimmerman, 2000; Díaz y Hernández, 2002; Lobato, 2006).

En la actualidad, se evalúa el uso de estas estrategias de autorregulación en el campo educativo de nivel superior, tomando en cuenta factores estratégicos, emocionales, motivacionales, temporales que los estudiantes usan en su proceso de aprendizaje (Cerna y Silva, 2018; Calderón,

2019; Bastacini y Kupczyszyn, 2020). Asimismo, ya se ha considerado el uso de estas actividades autorreguladoras como recursos ideales para optimizar las habilidades de comprensión de lectura que el discente universitario, posiblemente, debería haber desarrollado desde las etapas escolares (Pardo, 2015).

\section{Metacognición y estrategias de autorregulación.}

La metacognición guarda relación con aquellos saberes que el estudiante posee sobre los procesos cognitivos involucrados en el desarrollo de diferentes actividades tanto académicas como cotidianas, y se divide en el conocimiento y las experiencias metacognitivas, así como en las tareas y en las estrategias. La primera división refiere a la comprensión, por parte del estudiante, de la interacción de tres dimensiones: a. persona, cuando aquel se percatan de la existencia de diversos propios procesos cognitivos implicados en su propio proceso formativo personal y académico; $\mathrm{b}$. tarea, cuando aquel valora de forma significativa la información que recepciona; y c. estrategia, cuando aquel identifica las herramientas útiles para alcanzar el objetivo esperado en la realización de una determinada actividad académica, así como en qué tipo de proceso cognitivo utilizarlas. La 
segunda división y su reconocimiento logran que el discente estimule su pensamiento consciente al momento de solucionar problemas que se relacionan con otros problemas acontecidos con anterioridad; en otras palabras, aquel tiene la capacidad de activar estrategias que resultaron eficientes para una determinada situación (Flavell, 1979). Cabe resaltar que, según Paris y Winogrand (1990), citados en Pintrich, Wolters y Baxter (2000), ex metacognitivos.

De manera general y a partir de esta conceptualización sobre la metacognición, se puede determinar que el proceso en el que se desarrollan las estrategias del aprendizaje autorregulado amerita la activación de conocimientos manifestados por la metacognición y percibidos por el estudiante, quienes, finalmente, regulan sus diversos procesos cognitivos implicados en la ejecución de un proyecto académico, tal es el caso de razonar, solucionar problemas, atender, entre otros (Díaz y Hernández, 2002).

En ese mismo sentido, se puede señalar que aquellas estrategias, en el nivel superior, son concebidas a partir de la asociación entre la cognición, la metacognición y el aspecto contextual, puesto que requieren de procesos o procedimientos sencillos como repetir alguna información, así como de procedimientos más complejos como la reconstrucción de saberes previos, tomando en cuenta el uso del pensamiento crítico (Borgobello, Cattoni, Ventura, 2017).

\section{Competencia comunicativa y competencia discursiva en el marco educativo.}

La competencia comunicativa equivale a aquel conglomerado de destrezas adquiridas por el estudiante, enfocadas a la estructuración coherente de ideas al momento de utilizar de forma eficaz las reglas gramaticales y las reglas que constituyen los diferentes niveles lingüísticos: fonético, léxico, morfológico, semántico, normativo, entre otros, dentro de una comunidad lingüística, en la que los miembros de esta comparten un mismo dialecto; por ende, se cuidan los criterios de cuándo, qué, con quién, dónde y en qué forma expresarse oral y escrita (Hymes, 1971; Diccionario de términos clave de ELE, 2008).

Existen tres competencias que se interrelacionan y son dirigidas por la competencia comunicativa: la competencia lingüística, que guarda relación con la forma y el significado de las expresiones lingüísticas; por ende, la conforman categorías gramaticales como fonología, léxica, semántica, entre otras; la competencia sociolingüística, que considera los conocimientos, así como las habilidades sobre el uso de la lengua en la sociedad; por ello, toma en cuenta asuntos como marcadores lingüísticos de las relaciones sociales, convenciones de cortesía, el registro, las formas dialectales de la lengua, entre otros; y la competencia pragmática, que está constituida por los conocimientos de tres competencias más: la competencia discursiva, la competencia funcional y la competencia organizativa (MCER, 2018).

En el campo educativo, estas competencias enmarcadas dentro de la competencia comunicativa son adquiridas durante el proceso formativo del estudiante, en el que incluirá y optimizará la comunicación efectiva, aquella que se manifiesta cuando el discente produce de manera escrita u oral textos claros y persuasivos, que se adaptan a la situación (Morote y Labrador, s. f.). En el nivel universitario, estas competencias han sido evaluadas desde el modelo pedagógico basado en competencias, el cual atiende a los cambios constantes de la sociedad, a los valores, a los saberes y a las innovaciones; así como desde la perspectiva del propio estudiante frente al desarrollo de sus procesos discursivos y las capacidades que estos implican y del propio docente, quienes proponen, asertivamente, cambios en los planes de estudio para que no se pierda la conexión entre lo adquirido en las etapas iniciales de estudio y el resultado final después de haber egresado de la universidad (Domingo, Gallego, Rodríguez, 2013; Arnao y Medina, 2014; Núñez, 2016). 
Cabe resaltar que estos agentes de la enseñanza no siempre cuentan con una formación académica pertinente dentro del campo de la competencia comunicativa. Un claro ejemplo de ello se demostró en un estudio realizado en Cuba por Almanza, García y Jiménez (2019), en el que se descubrió que la mayoría de docentes de la Universidad de Camagüey desconocía cómo evaluar y cómo desarrollar la expresión escrita y oral de los futuros egresados.

En cuanto a la competencia discursiva, que forma parte de la competencia pragmática y esta, a su vez, de la competencia comunicativa, se puede señalar que se define como "la capacidad que posee el estudiante para diseñar, dirigir, estructurar textos, incluyendo aspectos genéricos como el desarrollo temático, y la coherencia y la cohesión, así como a la interacción, principios cooperativos y los turnos". (MCER, 2018, p. 139) En este artículo, se ha considerado, únicamente, la competencia discursiva escrita, aquella que se manifiesta en el uso de habilidades, por parte del estudiante, para permutar reglas de la gramática de una lengua en particular y utilizar indistintamente sus saberes sobre la tipología textual, a fin de expresar sus enunciados escritos de forma pertinente, tomando en cuenta el contexto; por ende, solo considera aspectos como el desarrollo temático y la coherencia y cohesión, señalados líneas más arriba (Diccionario de términos clave de ELE, 2008; Canale, 1983, citado en Borras-Comes, 2018).

Cabe destacar que estas capacidades que el estudiante adquiere cuando desarrolla su competencia discursiva escrita no deben desvincularse del aspecto social ni de las reglas que aquel debe reconocer y seguir, pues, el nivel de competencia comunicativa del usurario se diferenciará cuando, al momento de interactuar lingüísticamente con las demás personas, el discente manifieste determinados nivel educativo, clase social y matices afectivas (Quintanilla, 1999).

El desarrollo temático, que es considerado como una de las dimensiones de la competencia discursiva escrita, se manifiesta en las redacciones al momento de que el estudiante enlaza enunciados de forma lógica y clara, pues las mismas se organizan en torno a un tema en particular, el cual se va desarrollando progresivamente (Diccionario de términos clave de ELE, 2008; MCER, 2018). Ahora, la coherencia y cohesión, considerada como otra de las dimensiones de la competencia señalada líneas más arriba, refiere al sentido lógico que poseen las ideas al ser cohesionadas dentro de los párrafos que conforman el texto, sin dejar de lado el contexto (Calsamiglia y Tusón, 1999; Portolés, 2001).

Dentro de la educación, la competencia discursiva escrita, específicamente, es desatendida desde las etapas escolares, pues aquella queda relegada a los niveles medio y superior, en otras palabras, a los espacios universitarios (Pastor, 1994). Este hecho evidencia el escaso dominio de habilidades de redacción que los estudiantes presentan en el nivel superior, lo que le dificulta que estos continúen, acaso, de manera idónea, con su formación, puesto que, a lo largo del desarrollo de sus carreras, se enfrentan a situaciones, en las que deberán utilizar la forma académica del lenguaje para expresar sus ideas, así como construir sus conocimientos. En ese sentido, cuando el estudiante ingresa a la universidad, se podría suponer que ha adquirido un bagaje lingüístico y lectoescritor que le permitirá elaborar trabajos escritos empleando un discurso académico adecuado a su nivel. Hoy se sabe, a partir de las numerosas investigaciones realizadas, que existen carencias de nivel lingüísticodiscursivo en el discente universitario (Níkleva y Rico, 2016).

\section{La competencia discursiva en el texto académico.}

La competencia discursiva escrita considera las dimensiones de desarrollo temático y coherencia y cohesión, las cuales se manifiestan a través de la producción de textos. Por ende, dentro de estos, para la expresión eficaz de la primera dimensión, se toma en cuenta a aquellas normas propias y establecidas para los diferentes tipos de textos, puesto que cada uno de estos se encuentra 
cómo se desarrolla la temática a partir de los puntos o ideas de apoyo y de la intencionalidad del texto; por ejemplo, en el caso de un texto argumentativo, la intencionalidad se adjudica a la persuasión del lector (Sánchez y Vidal, s. f.). Del mismo modo, es ideal considerar la estructuración de las ideas argumentativas, las cuales refuerzan y sustentan las afirmaciones presentadas en la idea principal del texto (Iacona, 2018).

En cuanto a la coherencia y cohesión, que es la segunda dimensión de la competencia señalada líneas más arriba, dentro del texto, se considera el ordenamiento de estructuras lingüísticas de forma específica con la finalidad de que el mensaje sea interpretado adecuadamente por el receptor (Sorókina, 2008). También, contempla la normativa implícita en la coherencia, que permite que las ideas no se disocien del tema central del texto, así como aquellos recursos cohesivos que facilitan la concatenación de mensajes: la repetición, los conectores lógicos o marcadores discursivos y la elipsis (Rincón, 2009).

A partir de lo señalado, de forma panorámica, sobre el formato de un texto ideal, se puede indicar que el texto es un elemento autónomo con sentido lógico, que puede ser expresado oralmente o de manera escrita mediante la secuencia de componentes lingüísticos estructurados bajo las reglas de cohesión y coherencia (Van, 1993; Casado, citado en Martín y Montolío, 2008). Ahora, esta producción escrita, dentro del contexto universitario, es conocida como texto académico, que se distingue por lo siguiente: a. va dirigido a un público, cuyo lenguaje es formal; b. posee una configuración precisa; c. contiene testimonios que se sustentan en estudios realizados con anterioridad; d. debe respetar la normativa de la gramática de la lengua; e. debe ser entendible para el lector; y f. se distingue por cuatro intencionalidades en particular: que el texto pueda expresar los pensamientos e ideas, que convenza al receptor, que sea referencial o informativo y que no pierda la estética del contenido a través de una escritura con vocabulario cuidado (Kinneavy, 1969; Padrón, 1996; Bak, 2016).

Luego de deslindar las características del texto académico, se puede deducir que la competencia discursiva escrita se manifiesta en la redacción de estos escritos, los cuales son elaborados y considerados, a lo largo de las carreras universitarias de los discentes, como actividades académicas, que involucran diversos procesos de la cognición, adquiridos por aquellos durante el desarrollo de sus vidas, tales como seleccionar, organizar, integrar, atender y reflexionar sobre los saberes previos y saberes nuevos utilizados al momento de redactar (Roa, 2013).

\section{Estrategias de autorregulación y competencia discursiva escrita de discentes universitarios.}

A lo largo del proceso enseñanza-aprendizaje que se registra en el discente universitario, se observa que este no tiene la costumbre de planificar lo que va a proyectar en sus escritos, incluso, en reiterados casos, no revisa fuentes ni sus propios textos desde un punto de vista lógico, es decir, buscando el sentido y no solo la forma (Arias y García, 2006).

El estudiante de nivel superior debe reconocer que toda escritura pasa por 3 fases: planificación, desarrollo, revisión. La primera fase refiere a la elaboración de un borrador guía, en el que se incluyan los objetivos, la estructura, la temática y a quiénes va dirigido su texto. La segunda fase considera la iniciación de la redacción en sí y el control durante el desarrollo de esta, bajo criterios de jerarquización de contenidos. La tercera fase consiste en revisar reiteradas veces el proyecto final y, de haber algún error, buscar los recursos para subsanarlo (Roa, 2013).

Estas fases son destinadas a la producción eficiente de los mensajes inscritos en un texto como unidad autónoma; y, en el nivel superior, no solo se deberá considerar el significado de la producción escrita, sino, también, el sentido; desde ese punto, ambos se asocian y su adquisición deberá ser responsabilidad de la formación educativa universitaria, en la que son protagonistas tanto los agentes 
de enseñanza (Camps y Castelló, 2013). Estas características comprendidas en cada fase incluyen aspectos que se relacionan con los procesos cognitivos y metacognitivos, los mismo que se presentan en el uso de estrategias de autorregulación, pues estas se abastecen de los saberes metacognitivos para regular, finalmente, los procesos de la cognición que refieren a la finalidad de alcanzar con éxito el propósito del estudiante (Díaz y Hernández, 2002); en este caso, redactar un texto académico con un nivel efectivo de competencia discursiva escrita. Estas estrategias van a permitir que el educando pueda elaborar un esquema primario y orientador en cuanto a las estrategias y herramientas que van a ser utilizadas para el cumplimiento de sus metas, así como avizorar las expectativas frente la ejecución final de la tarea; asimismo, van a lograr que pueda cerciorarse de forma continua sobre el desarrollo, ejecución y estructuración de la tarea, así como la verificación del cumplimiento de las metas planteadas inicialmente; y, finalmente, van a permitir que realice juicios de valor sobre la ejecución general del proyecto, así como de la eficacia de los recursos de aprendizaje utilizados (Zimmerman, 2000; Díaz y Hernández, 2002; Lobato, 2006).

Ahora, que el discente utilice estrategias supone una previa meditación de acciones, las que van a encaminarlo, como ya se ha señalado, hacia la consecución de una meta concreta. En el caso de que sean autorreguladoras, tendrá la ventaja de seguir con un proceso jerarquizado y enfocado, en el que podrá gestionar las acciones del antes, del durante y del después de la actividad académica a desarrollar (redacción de textos), pues estas técnicas están conformadas por diferentes etapas, tales como planificación, planeación o forethought; supervisión, control o performance; y evaluación, revisión o self-reflection (Zimmerman, 2000; Díaz y Hernández, 2002; Lobato, 2006).

Se han realizado estudios en los que se demuestra la relación significativa entre diversas estrategias de aprendizaje y la redacción y, por ende, el desarrollo eficiente de la competencia discursiva escrita manifestada en esta, tal es el caso de las técnicas de aprendizaje basado en proyectos, las técnicas metacognitivas, las técnicas cognitivas y metacognitivas de producción de textos (Moreano y Roca, 2014; Llontop, 2015; Llontop, 2018). Dentro del contexto que se trata en este artículo, existen escasos estudios; sin embargo, la investigación llevada a cabo por Mesías (2018) ha demostrado la relación entre el uso de las 3 estrategias metacognitivas de aprendizaje, (planeación, monitoreo y evaluación), aquellas que equivalen a las estrategias del aprendizaje autorregulado, y el desarrollo de las competencias discursivas en el nivel superior.

Es necesario resaltar que las falencias tanto en el uso de estrategias autorreguladoras como en el desarrollo de la competencia discursiva escrita no solo es compromiso de los agentes de aprendizaje, sino, también, de los de enseñanza, pues, en muchas oportunidades, los primeros no dirigen adecuadamente sobre cómo redactar, qué procedimientos lingüístico-discursivos y cognitivos emplear en sus producciones textuales (Carlino, 2005; Roa, 2013), puesto que desconocen "los procesos cognitivos, efectivos y meta-cognitivos implicados en el aprendizaje significativo y, sobre todo, en su forma de enseñarlos”. (Díaz y Hernández, 2002, p. 233)

\section{Conclusiones}

Las estrategias de autorregulación, que están conformadas por etapas que se suceden de forma jerarquizada: planeación, control y evaluación, dentro del marco educativo de nivel superior, permiten que el discente universitario dirija la adquisición y construcción de sus propios aprendizajes (Zimmerman, 1989); y, actualmente, son estudiadas considerando criterios relacionados con las emociones, la gestión del tiempo, la motivación y las estrategias (Cerna y Silva, 2018; Calderón, 2019; Bastacini y Kupczyszyn, 2020). 
Se debe considerar aspectos cognitivos y metacognitivos implicados en el uso de estrategias de autorregulación, pues el discente universitario se valdrá de la activación de las experiencias y los conocimientos metacognitivos para regular los procesos cognitivos necesarios y que se interrelacionan para la ejecución de las diferentes actividades que se solicitan en el ámbito universitario (Díaz y Hernández, 2002), como la redacción de textos académicos.

La competencia discursiva escrita, la cual está incluida en la competencia comunicativa, dentro del marco educativo universitario, se manifiesta en la redacción de textos académicos, en los que el estudiante deberá demostrar la capacidad para estructurar y cohesionar ideas y pensamientos referidos a un tema central (MCER, 2018; Diccionario de términos clave de ELE, 2008). Sin embargo, no se puede dejar de mencionar que aquella manifiesta una desatención desde las etapas escolares y, más bien, su enseñanza es postergada hacia las etapas universitarias (Pastor, 1994). Este hecho se observa, en muchos casos, en el incipiente dominio de habilidades de redacción por parte de los agentes del aprendizaje.

Las fases por las que atraviesa la redacción de textos, conocidas como planificación, desarrollo y revisión (Roa, 2013), toman en cuenta aspectos relacionados con los procesos cognitivos y metacognitivos que se desarrollan también en el uso de las estrategias de autorregulación, pues estas se abastecen de los saberes metacognitivos para regular, finalmente, los procesos de la cognición implicados en alguna tarea (Díaz y Hernández, 2002). En el caso del desarrollo efectivo de la competencia discursiva escrita, manifestada en la redacción de textos, estas estrategias pueden convertirse en un recurso muy provechoso, pues, por sus características señaladas a lo largo de este artículo, permitirán que el discente universitario regule pertinentemente y de forma secuencial: antes, durante y después, su proceso de producción de textos escritos; pues, actualmente, existen estudios que abalan la relación significativa entre ambas variables de estudio de este trabajo de revisión, tal es el caso de Moreano y Roca (2014), Llontop (2015), Llontop (2018), Mesías (2018).

\section{Referencias}

Almanza, E., García, M., Jiménez, Y. (2019). El desarrollo y evaluación de la competencia discursiva del estudiante universitario: estrategia de capacitación para los docentes. Revista Transformación, 15(3), 342-353. http://scielo.sld.cu/scielo.php?script=sci_abstract\&pid=S2077-

$29552019000300342 \& \operatorname{lng}=$ es\&nrm=iso\&tlng=es

Arias, O., García, J. (2006). El papel de la revisión en los modelos de escritura. ICE-Aula Abierta, $88,37-52$.

Arnao, M., Medina, I. (2014). Percepción de los estudiantes de educación superior sobre el desarrollo de su competencia comunicativa. "UCV-HACER" Revista de investigación y cultura. 3(1). file:///C:/Users/user/Downloads/Dialnet-

PercepcionDeLosEstudiantesDeEducacionSuperiorSobre-5472522.pdf

Bak, N. (2016). Writing an academic text. University of Cape Town. 1-13. https://www.researchgate.net/publication/311846579

Bastacini, M., Kupczyszyn, K. (2020). Autorregulación en estudiantes universitarios: Estrategias de aprendizaje, motivación y emociones. Revista Educación, 44(1). https://www.scielo.sa.cr/scielo.php?pid=S2215-26442020000100327\&script=sci_arttext 
Borrás-Comes, J., Escobar, C. (2018). El desarrollo de la competencia discursiva en lengua extranjera en un entorno ICLES: La efectividad de la estrategia GUIDEWAY. https://www.researchgate.net/publication/317264989

Borgobello, A., Carttoni, M., Ventura, A. (2017). Aprendizaje autorregulado en el nivel universitario: Un estudio situado con estudiantes de psicopedagogía de diferentes ciclos académicos. Revista Electrónica Educare, 21(2), 1-20. https://www.redalyc.org/articulo.oa?id=194154995016

Calderón, J. (2019). Autorregulación del aprendizaje en estudiantes ingresantes a la universidad [tesis de pregrado, Pontificia Universidad Católica del Perú]. https://alicia.concytec.gob.pe/vufind/Record/PUCP_a4768c57c3683e1fca9d25a0dd13bc4f

Calsamiglia, H., Tusón, A. (1999). Las cosas del decir, Ariel, SA.

Camps, A., Castelló, M. (2013). La escritura académica en la universidad. Revista de Docencia Universitaria, 11(1), 17-36. https://polipapers.upv.es/index.php/REDU/article/view/5590

Carlino, P. (2005). La escritura en el nivel superior. En Escrib ir, leer y aprender en la universidad. Una introducción a la alfabetización académica. Buenos Aires: Fondo de Cultura Económica. https://www.aacademica.org/paula.carlino/3.pdf

Centro Virtual Cervantes: Diccionario de términos clave de ELE (2008). [versión en línea]. https://cvc.cervantes.es/ [Fecha de consulta: 30 de julio del 2020].

Cerna, C., Silva, M. (2018). Análisis del aprendizaje autorregulado en estudiantes universitarios. $\begin{array}{llll}\text { Revista Ciencia } \quad y \quad \text { Tecnología, } & \text { 16(1), }\end{array}$ https://www.researchgate.net/publication/316994961_El_aprendizaje_autorregulado_en_estu diantes_de_Educacion_Universitaria

Common european framework of reference for languages: learning, teaching, assessment. Companion volume with new descriptors (2018) [Marco Común Europeo de Referencia para las lenguas: aprendizaje, enseñanza y evaluación]. https://rm.coe.int/cefr-companion-volumewith-new-descriptors-2018/1680787989

Corbacho, A. (2006). Textos, tipos de texto y textos especializados. Revista de Filología, 77-90. https://www.dialnet.unirioja.es

Díaz, F., Hernández, G. (2002). Estrategias docentes para un aprendizaje significativo. Una interpretación constructivista. McGRAW-HILL.

Domingo, J., Gallego, J., Rodríguez, A. (2013). Percepción del profesorado sobre la competencia comunicativa en estudiantes de Magisterio. Perfiles educativos, 35(142), s. p. http://www.scielo.org.mx/scielo.php?script=sci_arttext\&pid=S0185-26982013000400005

Evangelista, D., Evangelista, E. (2019). La sintaxis en la competencia discursiva del estudiantado universitario de las áreas no humanísticas: Una propuesta pedagógica. Revista Actualidades $\begin{array}{llll}\text { Investigativas en } & \text { Educación, } & 19(2), & 1-22 .\end{array}$ https://revistas.ucr.ac.cr/index.php/aie/article/view/36902 
Flavell, J. (1979). Metacognition and cognitive monitoring. American Psychologist, 34(10), 906911. https://www.semanticscholar.org/paper/Metacognition-and-Cognitive-Monitoring\%3AA-New-Area-Flavell/ee652f0f63ed5b0cfe0af4cb4ea76b2ecf790c8d

Flórez, R. (1994). Hacia una pedagogía del conocimiento. McGraw-Hill.

García, Á., Fierro, B. (2017). La competencia analítico-textual/discursiva: un aprendizaje permanente. Atenas, 1(37), 31-46. https://www.redalyc.org/articulo.oa?id=478055147003

Hernández, A, Camargo, A. (2017). Autorregulación del aprendizaje en la educación superior en Iberoamérica: una revisión sistemática. Revista Latinoamericana de Psicología, 49(2), 146160 .

Hymes, D. (1971). Acerca de la competencia comunicativa. En Gómez J. (1996). Forma y función 9. Departamento de Lingüística Nacional de Colombia, 13-37.

Iacona, A. (2018). La argumentación. UAM, Unidad Cuajimalpa.

Kinneavy, J. (1969). Los objetivos básicos del discurso. College Composition and Communication, 20(5), 297-304.

Lobato, C. (2006). El estudio y trabajo autónomo del estudiante. En Métodos y modalidades de enseñanza centradas en el desarrollo de competencias. https://www.uaem.mx/sites/default/files/facultad-de-medicina/descargas/aprendizajeautodirigido.pdf

Llontop, M. (2015). Estrategias metacognitivas en la optimización del aprendizaje de los marcadores discursivos en estudiantes del programa de estudios básicos de la Universidad Ricardo Palma, año 2015. [Tesis doctoral, Universidad de San Martin de Porres]. http://repositorio.usmp.edu.pe/bitstream/handle/20.500.12727/1455/llontop_cmc.pdf?sequen $\mathrm{ce}=1$ \&isAllowed $=\mathrm{y}$

Llontop, M. (2018). Impacto de las estrategias del aprendizaje orientado en proyectos en la calidad de la redacción de textos funcionales en estudiantes universitarios. [Tesis de maestría, $\begin{array}{lllll}\text { Universidad de San } & \text { Martin }\end{array}$ http://repositorio.usmp.edu.pe/handle/20.500.12727/4058

Martín, M., Montolío, E. (2008). Los marcadores del discurso. Arco Libros.

Mesías, M. (2018). Las estrategias de aprendizaje metacognitivas y el desarrollo de las competencias discursivas-textuales en estudiantes universitarios, Callao, 2018 [tesis de doctorado, Universidad de San Martín de Porres]. http://repositorio.usmp.edu.pe/handle/usmp/4131

Moreano, R., Roca, M. (2014). Estrategias cognitivas y metacognitivas de composición escrita que se promueven en un curso de redacción en una universidad privada de Lima [tesis de maestría, $\begin{array}{llll}\text { Pontificia Universidad Católica } & \text { del }\end{array}$ http://tesis.pucp.edu.pe/repositorio/handle/20.500.12404/5771

Morote, P., Labrador, M. (s. f.). Competencia comunicativa en la universidad. https://cvc.cervantes.es/ensenanza/biblioteca_ele/aepe/pdf/congreso_50/congreso_50_42.pdf 
Níkleva, D., Rico, A. (2015). Análisis de la competencia lingüístico-discursiva escrita de los alumnos de nuevo ingreso del Grado de Maestro en Educación Primaria. Revista Signos. Estudios de Lingüistica, 40(90), 48-70. https://scielo.conicyt.cl/scielo.php?script=sci_arttext\&pid=S071809342016000100003

Núñez Cortés, J. A. (2016). El modelo competencial y la competencia comunicativa en la educación superior en América Latina. Foro de Educación, 14(20), 467-488. doi: http://dx.doi.org/10.14516/ fde.2016.014.020.023

Padrón, J. (1996). Análisis del discurso e investigación social. Temas para seminario. Caracas: Publicaciones del Decanato del Postgrado de la Universidad Nacional Experimental Simón Rodríguez.

Pardo, N. (2015). Estrategias autorreguladoras para la comprensión de textos académicos en los estudiantes universitarios. Revista Horizontes Pedagógicos, 17(2), 29-38. https://horizontespedagogicos.ibero.edu.co/article/view/17203

Pastor, S. (1994). El desarrollo de la competencia discursiva escrita del alumno de español, en V Congreso Internacional de ASELE, Montesa, S. y Gomis, P. (eds.). Tendencias actuales en la enseñanza del E/LE I. Málaga, Universidad de Málaga. https://cvc.cervantes.es/ensenanza/biblioteca_ele/asele/pdf/05/05_0245.pdf 